\title{
Consecutive negative findings on colonoscopy during surveillance predict a low risk of advanced neoplasia in patients with inflammatory bowel disease with long-standing colitis: results of a 15-year multicentre, multinational cohort study
}

\author{
Joren R ten Hove, ${ }^{1}$ Shailja C Shah, ${ }^{2,3}$ Seth R Shaffer, ${ }^{4}$ Charles N Bernstein, ${ }^{4}$ \\ Daniel Castaneda, ${ }^{2}$ Carolina Palmela, ${ }^{2}$ Erik Mooiweer, ${ }_{1}$ Jordan Elman, ${ }^{2}$ Akash Kumar, ${ }^{2}$ \\ Jason Glass, ${ }^{2}$ Jordan Axelrad, ${ }^{5}$ Thomas A Ullman, $^{2}$ Jean-Frederic Colombel, ${ }^{2}$ \\ Joana Torres, ${ }^{2,6}$ Adriaan A van Bodegraven, ${ }^{7,8}$ Frank Hoentjen, ${ }^{8,9}$ Jeroen M Jansen, 8,10 \\ Michiel E de Jong, ${ }^{8,9}$ Nofel Mahmmod, ${ }^{8,11}$ Andrea E van der Meulen-de Jong, ${ }^{8,12}$ \\ Cyriel Y Ponsioen, ${ }^{8,13}$ Christine J van der Woude, ${ }^{8,14}$ Steven H Itzkowitz, ${ }^{2}$ \\ Bas Oldenburg ${ }^{1,8}$
}

- Additional material is published online only. To view please visit the journal online (http://dx.doi.org/10.1136/ gutjnl-2017-315440).

For numbered affiliations see end of article.

Correspondence to Dr Bas Oldenburg, Department of Gastroenterology and Hepatology, University Medical Center Utrecht, 3584 CX Utrecht, The Netherlands; b.oldenburg@umcutrecht.nl

JRH and SCS contributed equally.

Received 10 October 2017 Revised 31 January 2018 Accepted 1 March 2018 Published Online First 2 May 2018

Check for updates

To cite: ten Hove JR Shah SC, Shaffer SR, et al. Gut 2019:68:615-622.

\section{ABSTRACT}

Objectives Surveillance colonoscopy is thought to prevent colorectal cancer (CRC) in patients with longstanding colonic IBD, but data regarding the frequency of surveillance and the findings thereof are lacking. Our aim was to determine whether consecutive negative surveillance colonoscopies adequately predict low neoplastic risk.

Design A multicentre, multinational database of patients with long-standing IBD colitis without high-risk features and undergoing regular CRC surveillance was constructed. A 'negative' surveillance colonoscopy was predefined as a technically adequate procedure having no postinflammatory polyps, no strictures, no endoscopic disease activity and no evidence of neoplasia; a 'positive' colonoscopy was a technically adequate procedure that included at least one of these criteria. The primary endpoint was advanced colorectal neoplasia (aCRN), defined as high-grade dysplasia or CRC.

Results of 775 patients with long-standing IBD colitis, 44\% ( $n=340)$ had $\geq 1$ negative colonoscopy. Patients with consecutive negative surveillance colonoscopies were compared with those who had at least one positive colonoscopy. Both groups had similar demographics, disease-related characteristics, number of surveillance colonoscopies and time intervals between colonoscopies. No aCRN occurred in those with consecutive negative surveillance, compared with an incidence rate of 0.29 to $0.76 / 100$ patient-years $(P=0.02)$ in those having $\geq 1$ positive colonoscopy on follow-up of 6.1 (P25-P75: 4.6-8.2) years after the index procedure.

Conclusion Within this large surveillance cohort of patients with colonic IBD and no additional high-risk features, having two consecutive negative colonoscopies predicted a very low risk of aCRN occurrence on followup. Our findings suggest that longer surveillance intervals in this selected population may be safe.

\section{Significance of this study}

What is already known about this subject?

- Because long-standing colonic IBD is associated with an increased risk of colorectal cancer (CRC), the current clinical recommendation is for interval colonoscopy for CRC surveillance.

- The recommended interval for CRC surveillance in this population ranges anywhere from 1 to 5 years, and most often every $1-2$ years, depending on clinical and endoscopic factors.

- Colonoscopic surveillance, which is associated with increased cost, patient inconvenience and small procedural risk, is of uncertain benefit in patients with no additional risk factors for CRC and well-controlled IBD with quiescent endoscopic disease on follow-up.

\section{INTRODUCTION}

Patients with long-standing colonic IBD are at an increased risk of developing colorectal cancer (CRC). ${ }^{12}$ Colonoscopic surveillance is widely used in these patients to prevent CRC-related mortality. Current guidelines use individual risk stratification to determine patient-specific surveillance intervals, ranging from 1 to 5 years. ${ }^{3-5}$ The European and British guidelines have incorporated a low-risk category for patients who have no high-risk or intermediate-risk features-such as primary sclerosing cholangitis (PSC), active inflammation or prior history of neoplasia-after an initial screening colonoscopy and allow for a 5-year surveillance interval in this selected population. This is in contrast to practice in North America, where there are no 
Significance of this study

What are the new findings?

- Using a large tri-national and multicentre database of patients with confirmed colonic IBD undergoing colonoscopic surveillance for CRC, we demonstrated that patients without high-risk demographics (eg, primary sclerosing cholangitis (PSC), personal or family history of colorectal neoplasia), undergoing adequate quality-metric surveillance colonoscopy without high-risk findings on examination, such as active inflammation, stricture, postinflammatory polyps and dysplasia (ie, a 'negative' colonoscopy), had a very low risk of future high-grade dysplasia or CRC.

- Of the cohort without high-risk features like PSC, no one with two consecutive negative surveillance colonoscopies developed high-grade dysplasia or CRC on follow-up.

\section{How might it impact on clinical practice in the foreseeable} future?

- Our findings inform current surveillance guidelines for the IBD population and suggest that in patients without additional risk factors for CRC and with at least two consecutive surveillance examinations showing endoscopically quiescent disease and no high-risk features, an interval longer than 2 years between surveillance examinations may be appropriate.

- A longer interval would optimise the cost and resource-tobenefit ratio of surveillance and would improve the quality of life for a large percentage of patients with IBD enrolled in surveillance.

distinct recommendations for the low-risk group, who are instead subjected to an every 1-year to 2-year colonoscopic surveillance protocol. This contributes a burden to healthcare systems in terms of costs and capacity, and to patients in terms of procedural risks, inconvenience and personal costs.

Several large well-designed studies in patients without IBD provide evidence-based guidance on the risk of CRC after negative investigations and inform clinical practice. ${ }^{6-10}$ For example, a recent study that included over 2100 average-risk patients found that an adequate quality-metric screening colonoscopy with negative results had significantly lower rates of adenomas or advanced neoplasms and no cancers at their 10-year follow-up colonoscopy, compared with people undergoing their first screening colonoscopy, with the authors concluding that a 10 -year interval for CRC screening after a negative colonoscopy is safe in the average-risk population. ${ }^{10}$ Furthermore, British screening colonoscopy guidelines suggest that after removal of sporadic adenomas, having two subsequent negative surveillance colonoscopies suggests that future examinations can be discontinued altogether. ${ }^{11}$ So far, data justifying longer surveillance intervals for low-risk patients with IBD, however, are lacking. We sought to address this question by using a multinational, multicentre database of patients with long-standing IBD colitis and no other highrisk features undergoing colonoscopic CRC surveillance. The overall aim was to determine whether two consecutive surveillance colonoscopies that demonstrated no intermediate-risk to high-risk findings (according to strict criteria) might predict absent or low rates of advanced colorectal neoplasia (aCRN), defined as high-grade dysplasia (HGD) or CRC, during follow-up.

\section{METHODS}

\section{Study population and case identification}

Patients undergoing colonoscopic surveillance for IBD colitis between 2000 and 2015 were retrospectively identified from three databases: a Dutch (Netherlands, NL) database that included two secondary and six tertiary centres, a US database that included one tertiary centre and a Canadian database that also included one tertiary centre. Cases were identified through a query of the electronic health record (EHR)-linked database for all cases of IBD or through the individual centre's patient registry.

\section{Patient selection (inclusion and exclusion criteria)}

After initial identification through the EHR query, individual charts were reviewed. Inclusion criteria were (1) diagnosis of IBD (UC, Crohn's disease (CD), IBD undifferentiated (IBD-U)) with colonic involvement confirmed by pathology, (2) confirmed colonic disease duration of at least 8 years (referred to hereafter as 'long-standing'), (3) enrolment in a dysplasia surveillance programme, (4) at least two separate colonic pathology reports from surveillance examinations, (5) at least left-sided disease extent (UC or IBD-U) or involvement of $>30 \%$ of the colonic surface (CD or IBD-U) and (6) no aCRN prior to, or at, index colonoscopy during the defined study period. Exclusion criteria were (1) CD without colonic involvement, (2) UC or IBD-U with limited proctitis and CD or IBD-U with $<30 \%$ colonic involvement and (3) less than two surveillance colonoscopies with available pathology. So-called 'high risk' patients were also excluded if they had any of the following a priori determined factors: PSC, history of any dysplasia or CRC prior to, or at, index colonoscopy, prior history of stricture or first-degree relative with a history of CRC.

Date of enrolment was the date of the first surveillance colonoscopy within the stated study period (hereafter referred to as the 'index colonoscopy'). Surveillance procedures were defined as colonoscopies in which either segmental random biopsies or chromoendoscopy were employed. We excluded subjects in whom the index or subsequent colonoscopy had inadequate bowel preparation according to the endoscopists' overall impression and/or caecal intubation was not achieved, as these procedures are considered technically insufficient for surveillance. We also excluded any colonoscopies that were performed for an indication other than surveillance, for example, medically refractory disease, or those that were performed with surveillance as the indication but did not employ at least segmental biopsies or chromoendoscopy. Thus, we only included patients who had at least two consecutive surveillance colonoscopies with adequate quality metrics, followed by at least one mode of pathological assessment on subsequent follow-up-either another surveillance colonoscopy, a colonoscopy for medical refractory disease where biopsies were taken or colectomy (segmental, subtotal, total) - to determine whether or not there was an eventual diagnosis of dysplasia and/or CRC.

Any interval aCRN diagnosis, irrespective of diagnostic setting (e.g, colectomy), was recorded.

\section{Data collection}

The following baseline demographic and clinical data were abstracted: date of birth, sex, age of IBD diagnosis, IBD type (UC, CD or IBD-U), maximum extent of colonic disease at any time during follow-up, family history of CRC and diagnosis of PSC by endoscopic retrograde cholangiopancreatography (ERCP) and/or magnetic resonance cholangiopancreatography 


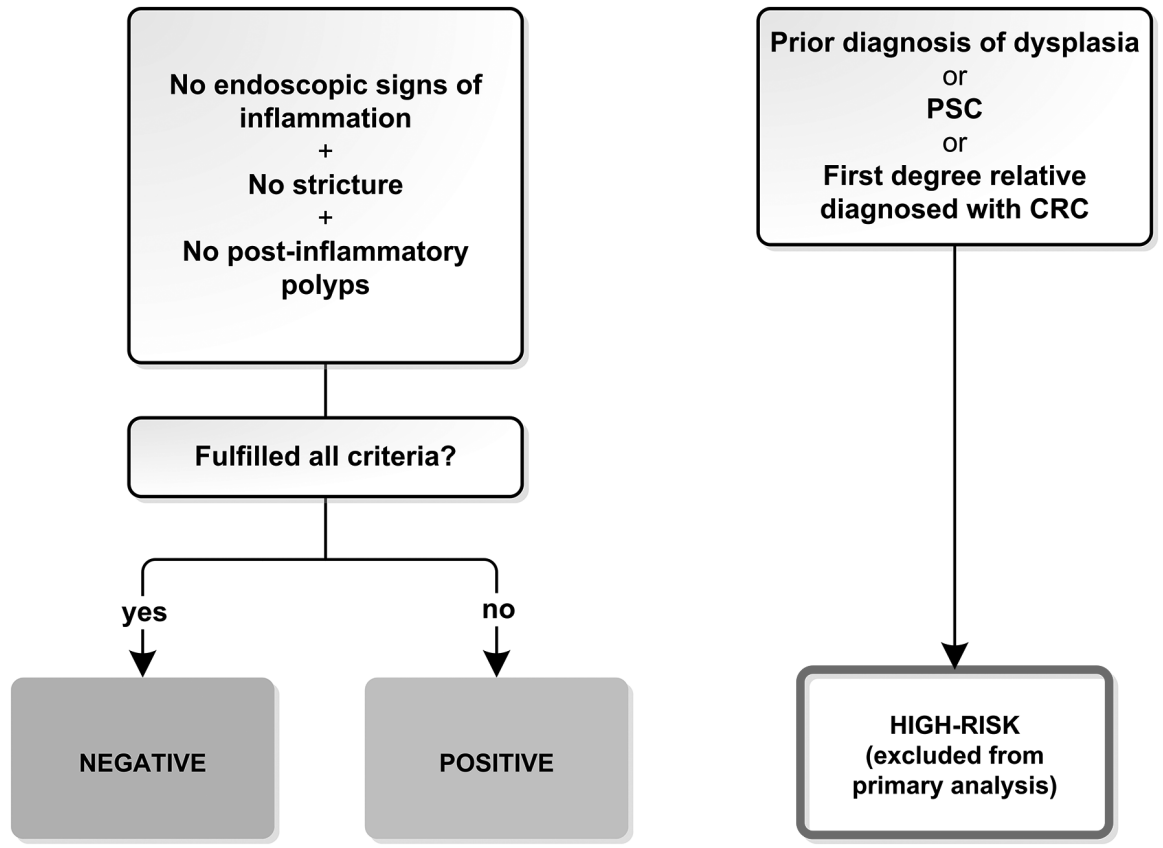

Figure 1 Group allocation algorithm (index procedure). All colonoscopic procedures (positive or negative) had caecal intubation and adequate bowel preparation. CRC, colorectal cancer; PSC, primary sclerosing cholangitis.

(MRCP). Medication exposure was defined as duration of use for at least 3 months according to EHR documentation and was recorded for 5 -aminosalicylates, immunomodulators (azathioprine or 6-mercaptopurine) and biologics.

Details of the index surveillance colonoscopy and each subsequent surveillance examination were recorded, including quality of bowel preparation as determined by the endoscopist at the time of the examination (adequate (excellent or good) or inadequate (fair or poor), the latter of which were excluded), use of chromoendoscopy, most proximal colonic extent examined (with exclusion of those procedures where caecal intubation was not achieved), overall impression of endoscopic inflammation (none or remission, mildly active, moderately active, severely active), presence of postinflammatory polyps ('pseudopolyps'), stricture(s) and visible lesions. Lesion location was categorised as left-sided if located distal to the splenic flexure and right-sided if located proximal to the splenic flexure. The number of surveillance colonoscopies, the time interval between examinations and the duration of active follow-up with the treating gastroenterologist were recorded. A standard data collection form was used at all centres.

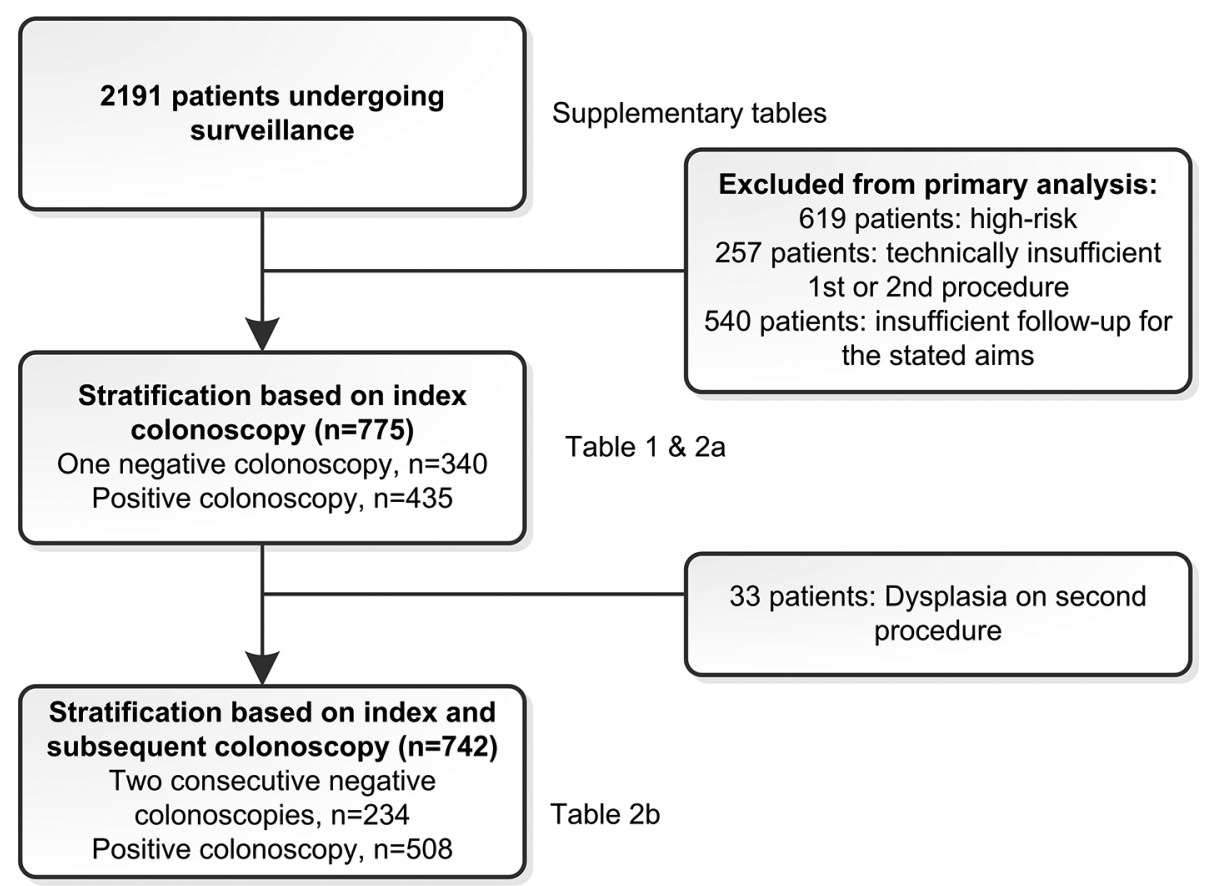

Figure 2 Selection of patients and group allocation. 


\section{Histological data}

All histological diagnoses were recorded as detailed in the original pathology report; no specimens were re-reviewed or altered for the purpose of this study. Dysplasia was reported as indefinite (IND), low-grade (LGD) or high-grade (HGD). For this study, any lesion graded as LGD or higher was defined as neoplasia; aCRN included any HGD or CRC. IND was categorised as non-neoplastic. Of note, it is routine practice at all institutions participating in this study that a specimen concerning for neoplasia (including indefinite dysplasia) be reviewed by two pathologists and consensus reached before final reporting in the EHR.

\section{Stratification of index colonoscopies}

Patients who met inclusion criteria were first assigned to one of two groups based on their initial surveillance colonoscopy within the studied timeframe-either the negative (NEG) index colonoscopy group or the positive (POS) index colonoscopy groupand then subsequently categorised according to findings on their second surveillance colonoscopy, as either NEG-NEG ('double negative') if the two consecutive colonoscopies were negative (as defined below) or NEG-POS, POS-NEG or POS-POS if the index and/or consecutive colonoscopies included any of the positive findings as detailed.

For this study, a 'negative' colonoscopy was strictly defined as a completed surveillance colonoscopy with no postinflammatory polyps, no strictures, absence of any endoscopic disease activity and no dysplasia (figure 1). If any of these criteria was not met, the colonoscopy was considered 'positive'. If neoplasia (LGD or higher) was identified on the surveillance procedure following the index procedure ('second colonoscopy', figure 2), this was recorded, but these patients were excluded from the primary analysis assessing the subsequent development of neoplasia following two surveillance procedures. If the diagnosis was LGD, these patients were instead allocated to the high-risk group and analysed separately; if the diagnosis was aCRN, these patients were excluded completely since the primary outcome had been reached (see below). As noted, patients deemed to be at high risk for dysplasia and CRC at the outset (eg, concomitant PSC, prior history of dysplasia, family history of CRC) were excluded from the primary analysis, but were allocated to the high-risk group and analysed separately (see below).

\section{Primary and secondary outcomes}

The primary outcome was occurrence of aCRN following consecutive surveillance colonoscopies after the index examination. Secondary outcomes included any neoplastic diagnosis during follow-up (ie, LGD or higher), and a diagnosis of aCRN following any two negative surveillance examinations during the follow-up period. Patients were censored at the time of aCRN diagnosis, colectomy, the date of the last gastroenterology follow-up or the end of the predetermined study interval, whichever occurred first.

\section{Statistical analysis}

Descriptive statistics were generated for patients meeting the inclusion criteria. Chi-square test was used for categorical variables and Student's t-test for continuous variables. The primary and secondary outcomes were analysed using a time-to-event analysis and Kaplan-Meier survival curves were generated to compare the various groups according to their surveillance colonoscopy findings. The incidence rate of neoplasia and aCRN was reported as the number of cases per 100 patient-years of follow-up (pty) with $95 \%$ CI. The 25 th to 75 th percentiles were reported as P25-P75. Total follow-up time was reported as the time of index colonoscopy until the first censored time point. Because patients were grouped based on their findings of each of their two consecutive colonoscopies, group allocation was ultimately determined at the time of the second colonoscopy; thus, we also reported a second follow-up interval for each of the analyses, which is the time from the date of the second colonoscopy until the first censored time point. All statistical analyses were performed using SPSS V.22 (IBM).

\section{Study oversight}

The institutional review board for each of the participating sites approved the creation and analysis of a longitudinal retrospective

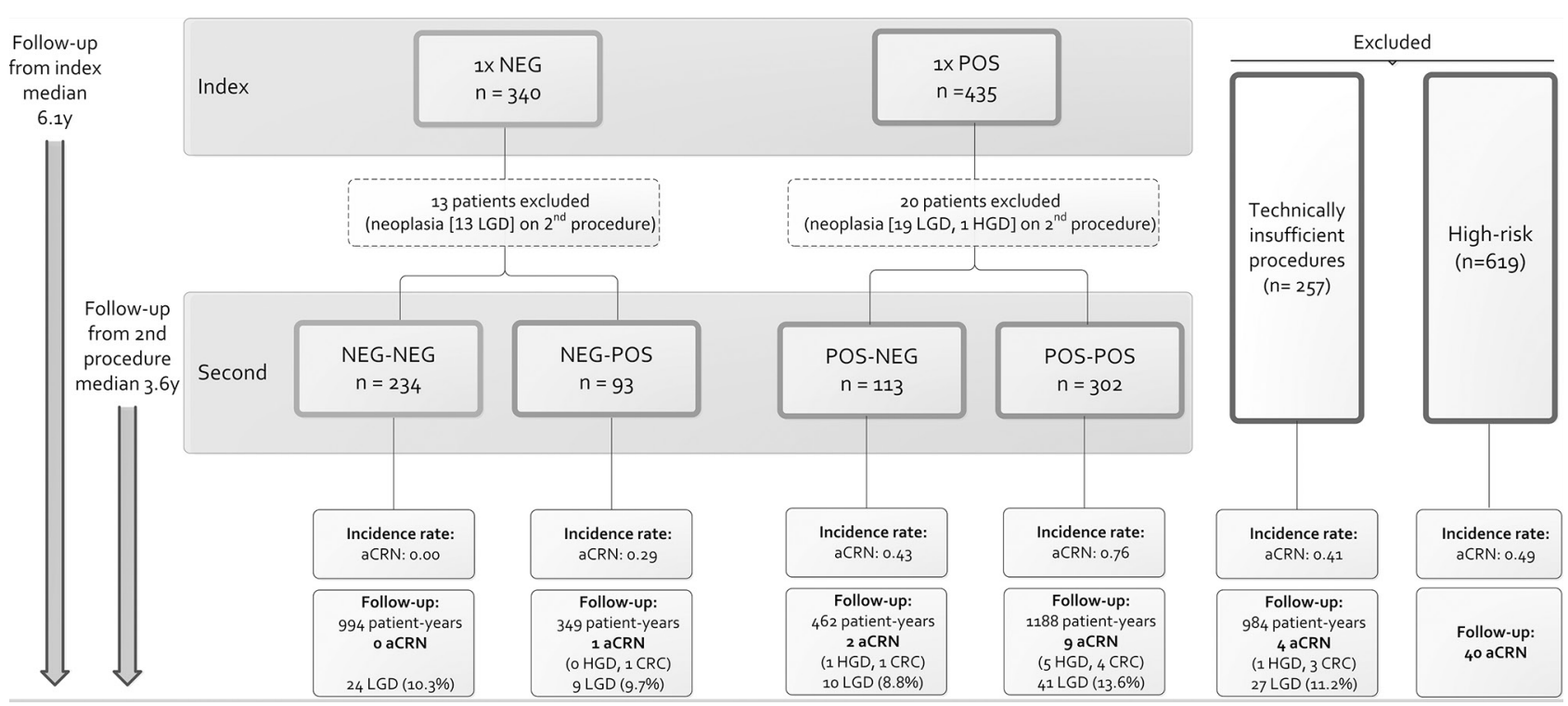

Figure 3 Incidence rate of advanced colorectal neoplasia (aCRN) on follow-up after group allocation. CRC, colorectal cancer; HGD, high-grade dysplasia; LGD, low-grade dysplasia; NEG, negative colonoscopy; POS, $\geq 1$ high-risk feature on colonoscopy. Incidence rate is reported per 100 patientyears. 
Table 1 Demographic, clinical and endoscopic characteristics of combined cohort $(n=775)$

\begin{tabular}{|c|c|}
\hline Variable & Value \\
\hline Male & $363(46.8 \%)$ \\
\hline Age at index colonoscopy, years, median (P25-P75) & $44(35-53)$ \\
\hline \multicolumn{2}{|l|}{ IBD diagnosis } \\
\hline UC & $474(61.2 \%)$ \\
\hline Crohn's colitis & $280(36.1 \%)$ \\
\hline Indeterminate colitis & $21(2.7 \%)$ \\
\hline Disease duration, years, median (P25-P75) & $13(9-21)$ \\
\hline \multicolumn{2}{|l|}{ Maximum disease extent (endoscopic) } \\
\hline Extensive/pancolitis & $390(50.3 \%)$ \\
\hline Left-sided/ $>30 \%$ colonic involvement & $322(41.6 \%)$ \\
\hline Not specified & $63(8.1 \%)$ \\
\hline \multicolumn{2}{|l|}{ Medication exposure } \\
\hline 5-Aminosalicylate & $658(84.9 \%)$ \\
\hline Immunomodulator & $386(52.0 \%)$ \\
\hline Biological & $176(22.7 \%)$ \\
\hline Negative index colonoscopy* & $340(43.9 \%)$ \\
\hline $\begin{array}{l}\text { Duration of follow-up after index colonoscopy, years, median } \\
\text { (P25-P75) }\end{array}$ & $6.1(4.6-8.2)$ \\
\hline
\end{tabular}

*Defined as a technically adequate surveillance colonoscopy with no postinflammatory polyps, no strictures, absence of any endoscopic disease activity and no evidence of neoplasia (low-grade dysplasia or higher) in either random or targeted biopsies

cohort database of patients with colonic IBD undergoing colonoscopy for CRC surveillance. This study was performed in compliance with the Health Insurance Portability and Accountability Act guidelines.

\section{RESULTS}

The baseline characteristics for the 775 patients with longstanding IBD colitis undergoing colonoscopic surveillance meeting initial inclusion criteria (figure 3 ) are detailed in table 1 , with characteristics of each of the individual cohorts from the NL, USA and Canada detailed in online supplementary table 1 . The cohort comprised 363 men $(47 \%)$ with a median age at study entry of 44 (P25-P75: 35-53) years. The most frequent IBD type was UC $(n=474 ; 61 \%)$ with median disease duration of 13 (P25-P75: 9-21) years.

The index colonoscopy (ie, first surveillance colonoscopy within the study period) fulfilled all predefined criteria for a negative examination in 340 patients (44\%) with all others classified as positive examinations. Both groups were similar in terms of demographics and disease characteristics including age at index colonoscopy, sex, IBD type and disease duration, as well as the number of subsequent surveillance colonoscopies after the index procedure (3.9 vs $3.9, \mathrm{P}=0.76$ ). In general, medication use was higher in the groups with a positive index colonoscopy. The interval between the index colonoscopy and the subsequent surveillance examination was 2.2 years for both groups $(\mathrm{P}=0.66)$. Additional characteristics of the two groups stratified according to the findings on the index and subsequent surveillance procedures are detailed in table $2 \mathrm{~A}$ and $\mathrm{B}$, respectively. The duration of follow-up for the cohort following the index surveillance procedure was 6.1 (P25-P75: 4.6-8.2) years.

\section{Primary outcome}

The frequency of aCRN cases on follow-up after a negative index colonoscopy was $1 / 340(0.3 \%)$ compared with $12 / 435$ $(2.8 \%)$ in those with a positive index colonoscopy. Patients

Table 2 Baseline characteristics after stratification based on first (2A) and second (2B) surveillance colonoscopies

\begin{tabular}{|c|c|c|c|c|c|c|}
\hline & \multicolumn{3}{|l|}{$2 \mathrm{~A}$} & \multicolumn{3}{|l|}{ 2B } \\
\hline & $\begin{array}{l}\text { Negative index } \\
\text { colonoscopy }(n=340)\end{array}$ & $\begin{array}{l}\text { Positive index } \\
\text { colonoscopy }(n=435)\end{array}$ & $P$ value & $\begin{array}{l}\text { Double negative } \\
(n=234)\end{array}$ & $\begin{array}{l}\text { Any positive } \\
\text { colonoscopy }(n=508)\end{array}$ & $P$ value \\
\hline Male (\%) & $151(44.4 \%)$ & $212(48.7 \%)$ & 0.23 & $100(42.7 \%)$ & $247(48.6 \%)$ & 0.14 \\
\hline $\begin{array}{l}\text { Age at index colonoscopy, years, median (P25- } \\
\text { P75) }\end{array}$ & $45(36-53)$ & $43(33-53)$ & 0.17 & $46(37-54)$ & $44(34-53)$ & 0.12 \\
\hline \multicolumn{7}{|l|}{ IBD diagnosis } \\
\hline Ulcerative colitis & $206(60.6 \%)$ & $268(61.6 \%)$ & 0.72 & $140(59.8 \%)$ & $314(61.8 \%)$ & 0.87 \\
\hline Crohn's colitis & $123(36.2 \%)$ & $157(36.1 \%)$ & & $87(37.2 \%)$ & $180(35.4 \%)$ & \\
\hline Indeterminate colitis & $11(3.2 \%)$ & $10(2.3 \%)$ & & $7(3.0 \%)$ & $14(2.8 \%)$ & \\
\hline $\begin{array}{l}\text { Disease duration at index colonoscopy, years, } \\
\text { median (P25-P75) }\end{array}$ & $14(9-22)$ & $13(9-20)$ & 0.33 & $14(9-23)$ & $13(9-20)$ & 0.29 \\
\hline \multicolumn{7}{|l|}{ Medication exposure } \\
\hline 5-Aminosalicylate & $278(81.8 \%)$ & $380(87.4 \%)$ & 0.03 & $190(81.2 \%)$ & $439(86.4 \%)$ & 0.07 \\
\hline Immunomodulator & $160(47.1 \%)$ & $239(54.9 \%)$ & 0.03 & $110(47.0 \%)$ & $276(54.3 \%)$ & 0.06 \\
\hline Biological & $54(15.9 \%)$ & $122(28.0 \%)$ & $<0.01$ & $33(14.1 \%)$ & $137(27.0 \%)$ & $<0.01$ \\
\hline Postinflammatory polyps & - & $176(40.5 \%)$ & - & - & $167(32.9 \%)$ & - \\
\hline \multicolumn{7}{|l|}{ Endoscopic inflammation } \\
\hline Remission/inactive & $340(100 \%)$ & $117(26.9 \%)$ & - & $234(100 \%)$ & $202(39.8 \%)$ & - \\
\hline Mild & - & $238(54.7 \%)$ & & - & $231(45.5 \%)$ & \\
\hline Moderate & - & $63(14.5 \%)$ & & - & $59(11.6 \%)$ & \\
\hline Severe & - & $17(3.9 \%)$ & & - & $16(3.1 \%)$ & \\
\hline \multicolumn{7}{|l|}{ Duration of follow-up, years, median (P25-P75) } \\
\hline After index colonoscopy & $6.3(4.8-8.6)$ & $6.1(4.4-8.1)$ & 0.12 & $6.3(4.9-8.7)$ & $6.0(4.4-8.1)$ & 0.05 \\
\hline After second colonoscopy & $3.8(2.2-5.4)$ & $3.5(2.1-5.3)$ & 0.24 & $4.0(2.3-5.6)$ & $3.4(2.1-5.3)$ & $<0.01$ \\
\hline Total colonoscopies during follow-up, mean & 3.9 & 3.9 & 0.76 & 3.9 & 4.0 & 0.71 \\
\hline $\begin{array}{l}\text { Time between index and second colonoscopy, } \\
\text { median, years (P25-P75) }\end{array}$ & $2.2(1.9-3.1)$ & $2.2(1.6-3.0)$ & 0.66 & $2.2(1.9-3.1)$ & $2.2(1.7-3.0)$ & 0.94 \\
\hline
\end{tabular}




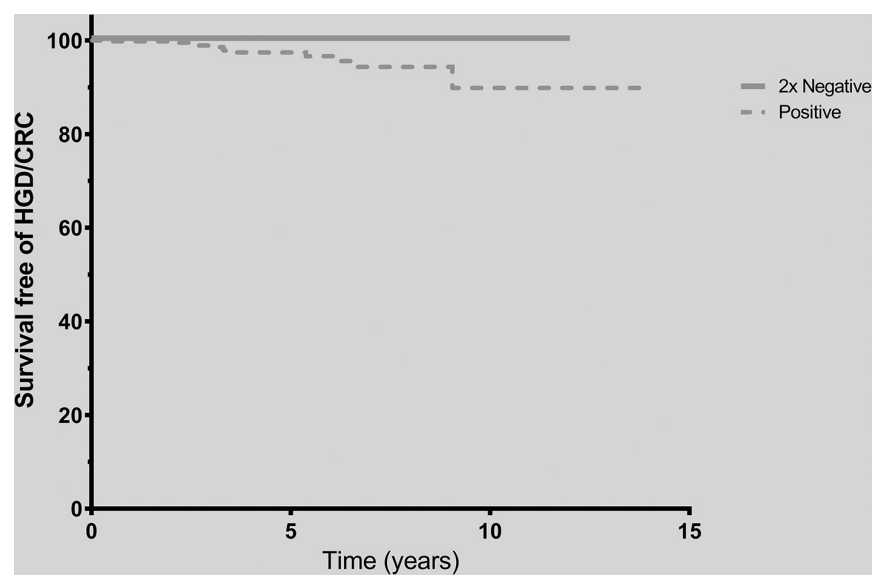

Figure 4 Incidence rate of aCRN according to consecutive surveillance colonoscopic findings (double negative vs at least one positive). Logrank test, $\mathrm{P}=0.01$. CRC, colorectal cancer; $H G D$, high-grade dysplasia.

were further stratified according to the findings on the subsequent surveillance colonoscopy (figure 2). The incidence rate of aCRN in those with double-negative surveillance examinations was $0 / 100$ pty compared with $0.29-0.76 / 100$ pty in those with at least one positive examination $(\mathrm{P}=0.01, \log$-rank test, figure 4$)$. That is, no cases of aCRN developed over the follow-up period in those patients with a negative index colonoscopy and negative subsequent surveillance colonoscopy (NEG-NEG). Incidence for the subgroups were NEG-POS 0.29/100pty $(95 \% \mathrm{CI}$ 0.01 to 1.41$)$, POS-NEG $0.43 / 100$ pty $(95 \%$ CI 0.07 to 1.43 ) and POS-POS $0.76 / 100$ pty $(95 \%$ CI 0.37 to 1.39 ). The time interval between the index surveillance colonoscopy and subsequent surveillance examination was the same between both the double-negative group and the group with at least one positive colonoscopy (median 2.2 years), as was the number of surveillance colonoscopies performed during the follow-up period. The frequencies of colectomies for the subgroups (NEGNEG, NEG-POS, POS-NEG, POS-POS) were 8/234 (3.4\%), $6 / 93$ (6.5\%), 5/113 (4.4\%) and 18/302 (6.0\%), respectively $(\mathrm{P}=0.51)$. The median follow-up time following the second surveillance procedure for the cohort was 3.6 years (P25-P75: $3.6-5.4$ years).

\section{Secondary outcomes}

Of interest, there were 91 additional patients who had two consecutive negative colonoscopies at any time during the study period but not including the index examination closest to the start of the predetermined study period (for example, a patient with a positive colonoscopy in 2000 at enrolment (POS index colonoscopy) who had negative examinations according to the predefined criteria in 2005 and 2007). Importantly, none of these patients developed aCRN over the period of follow-up (data not shown).

Among patients in whom the index and/or subsequent surveillance procedure was technically insufficient $(n=257)$ and thus precluded them from the primary analysis, four cases of aCRN occurred during the follow-up period, with an incidence rate of $0.41 / 100$ pty.

All four groups (NEG-NEG, NEG-POS; POS-NEG; POS-POS) were found to have LGD during subsequent follow-up, but there were no significant differences between the groups with respect to frequency and incidence rate $(\mathrm{P}=0.68)$ (figure 2$)$.

\section{Inclusion of high-risk patients}

We performed a sensitivity analysis in which we included the 619 patients who were excluded from the primary analysis either at the outset of the study because of predefined high-risk features $(\mathrm{n}=587)$ - that is, PSC, history of any dysplasia prior to, or at, index colonoscopy, prior history of stricture, or first-degree relative with a history of CRC (online supplementary tables 2,3)—or because of a diagnosis of LGD prior to their second colonoscopy $(n=32)$. Only one patient developed aCRN following the index colonoscopy and was excluded from the analysis completely (figure 2). The overall rate of aCRN in this extended cohort over the follow-up period was $0.49 / 100$ pty. Although the overall incidence rates for aCRN were higher compared with the selected cohort for the primary analysis, the previously observed differences in incidence rates remained significantly different (online supplementary figure 1). The incidence rates for the subgroups were NEG-NEG $0.15 / 100$ pty (95\% CI 0.03 to 0.51 ), NEG-POS $0.44 / 100$ pty $(95 \%$ CI 0.07 to 1.45 ), POS-NEG $0.65 / 100$ pty (95\% CI 0.21 to 1.56 ) and POS-POS 0.73 (95\% CI 0.38 to 1.27). As expected, those in this extended group who were allocated to the double-negative group based on two consecutive negative surveillance colonoscopies had the lowest incidence of aCRN when compared with those with either examination labelled positive, although not 0/100pty as in the primary analysis that excluded this 'high-risk' cohort.

\section{DISCUSSION}

In this well-characterised, multinational and multicentre database of patients with long-standing IBD colitis undergoing routine CRC surveillance, we found markedly low rates of subsequent aCRN diagnoses in patients with a negative index examination according to predefined criteria. The incidence rate of aCRN after a single negative surveillance colonoscopy was already low at $0.09 / 100$ pty, and this rate dropped to zero in those patients with two consecutive negative surveillance examinations during the follow-up period. Moreover, this pattern and its statistical significance were also seen when we analysed the extended cohort that included patients with any high-risk characteristics for aCRN (eg, PSC, history of any neoplasia prior to, or at, index colonoscopy, prior history of stricture or first-degree relative with a history of (RC) or a diagnosis of LGD following the index procedure. Our findings suggest that, at least in the population with long-standing IBD colitis and otherwise no additional high-risk features, a CRC surveillance interval longer than 2 years is safe following two negative colonoscopies. Our predetermined definition of a negative colonoscopy was strict, yet it was based on readily available parameters recommended in current guidelines. ${ }^{3} 12$

While patients with IBD colitis are considered to carry a higher-than-average CRC risk, estimated at 5\% after 20 years disease duration, ${ }^{2}$ the appropriateness of the recommended intervals of colonoscopic surveillance in this population has been incompletely investigated. Considering that the overall risk of CRC in IBD is decreasing over time ${ }^{2} 13$ and the sensitivity for detecting dysplasia is increasing due in part to technological advancements in neoplasia detection, ${ }^{14}$ if surveillance is maintained at overly frequent intervals in all patients with IBD, IBD surveillance programmes are at risk of becoming high-intensity/low-value cancer prevention strategies. European guidelines advocate a risk stratification model when determining appropriate surveillance intervals, but recommendations are inconsistent geographically and the data corroborating such recommendations are limited. ${ }^{3515}$ Data on incidence, test sensitivity, natural history 
and sojourn time of precursor lesions are essential to optimise the current surveillance strategies.

We designed our risk stratification model for the present study according to current British and European guidelines, which recommend allocating patients undergoing IBD surveillance into one of three groups ${ }^{315}$-low, intermediate and high-risk-each with distinct recommended surveillance intervals. We further made our definition of a 'negative' colonoscopy strict in order to truly define a lower risk IBD population, but also to maximise use of objective measures to enhance clinical reproducibility and facility in a practice-based setting. Patient characteristics including PSC, ${ }^{16} 17$ family history of $\mathrm{CRC}^{18}$ and personal history of neoplasia, ${ }^{19}$ as well as endoscopic features including strictures, ${ }^{20}$ inflammation, ${ }^{21} 22$ and postinflammatory polyps ${ }^{20} 2324$ have all been shown to increase the risk of subsequent neoplastic transformation in IBD colitis; thus, complete absence of these features was necessary to meet criteria for a negative investigation. Based on these criteria, less than $30 \%$ of patients in our database had high-risk demographics that necessitated yearly surveillance colonoscopies. That said, the risk associated with postinflammatory polyps may vary according to the density and extent of postinflammatory polyps and depends ultimately on how completely surveillance can still be performed. More research is required to understand the true risk that postinflammatory polyps pose, be it direct (by neoplastic transformation of the polyp itself) or indirect (due to hindered visualisation of other neoplastic polyps). While there are emerging data suggesting that postinflammatory polyps are not independently associated with an increased risk of neoplasia, we chose to err on the side of conservative and categorised an endoscopic finding of postinflammatory polyps as a positive colonoscopy. Among the remaining low-risk patients, over one-third had at least one negative surveillance colonoscopy and underscores the potential cost-saving and resource-saving, not to mention patient convenience implications, if surveillance intensity can be downgraded for this select population.

The definition of a low-risk surveillance population for risk stratification purposes in our study also has biological credence. Patients without signs of previous or ongoing inflammation have acquired less cumulative inflammatory damage over time. A decreased mutational burden translates into a decreased risk of developing aCRN. ${ }^{25}$ Moreover, endoscopically active disease, strictures and postinflammatory polyps all affect visibility during colonoscopy and thus the sensitivity of the colonoscopy itself for dysplasia and CRC detection. Having two strictly defined negative consecutive colonoscopies with enhanced diagnostic sensitivity broadly reduces the likelihood of missed dysplasia and optimises the negative predictive value of colonoscopy in clinical practice.

While our data raise the questions of whether (1) we may rely on the ability of two consecutive negative surveillance colonoscopies to adequately predict sustained low aCRN risk and, accordingly, (2) whether we may safely prolong surveillance intervals, it is important to bear two caveats in mind when interpreting our data. First, although we attempted to maximise follow-up times, we had insufficient data to look at the aCRN risk beyond 5 years. Second, in the subgroup of patients with two consecutive negative colonoscopies, LGD was nonetheless detected in $11 \%$ of patients during follow-up, thus implying that there is still a role for ongoing surveillance in these patients.

As a contextual comparison, in the non-IBD background population, the lifetime risk of developing CRC is approximately $4.5 \%$, and is subject to regional and temporal variations. ${ }^{26}$ For patients with IBD, the risk of CRC is broadly estimated to be increased by a factor of 2 , although numbers vary according to the population studied. ${ }^{27}$ Nevertheless, caution should be exercised when comparing neoplasia outcomes between patients with IBD and without IBD, particularly since the trajectory of dysplasia progression is, generally speaking, more rapid in the former and implicates nuances in the dysplasia-carcinoma sequence. ${ }^{19}$ Indeed, CRC screening intervals for patients without IBD may safely extend up to 10 years, far more prolonged than the currently recommended screening intervals in patients with long-standing IBD colitis. ${ }^{828} 29$

Our study has some limitations, in addition to those inherent to any retrospective analysis. First, although our duration of follow-up was sufficient for achieving our study aims, we are unable to reliably comment on the risk of aCRN after 5 years. Second, the index colonoscopy was defined as the first surveillance examination within our predefined study period and thus may not represent the true index surveillance colonoscopy in patients' IBD courses. That said, this may better represent the real-world scenario and enhance the applicability of our findings since two negative consecutive colonoscopies may occur at any point during patients' surveillance programme. Thirdly, because our database was strictly limited to surveillance colonoscopies, we did not specifically investigate whether intermittent flares between surveillance examinations significantly affect the neoplasia risk. Our study was also not designed to comment on the optimal screening interval for this low-risk group, nor of the necessity of concomitant clinical remission. Lastly, because chromoendoscopy was not routinely available at the included institutions for most of the included study period, we were unable to evaluate its adjunctive role in defining a negative colonoscopy and subsequent neoplastic risk.

There are several strengths to our study. By predefining variables and strict selection criteria, we were able to combine three large cohorts of patients with confirmed IBD colitis from three different countries undergoing CRC surveillance without compromising detailed documentation of patients' baseline demographics, disease-related characteristics, and follow-up colonoscopic and histological findings. In this way, we maximised generalisability, sample size and power, while still precisely and reliably categorised patients according to our predetermined criteria.

In conclusion, in this large multicentre, multinational cohort of well-characterised patients with IBD colitis undergoing active CRC surveillance, we identified that in a selected low-risk group, having two consecutive negative surveillance examinations predicts a very low and potentially negligible risk of aCRN on continued follow-up. Our findings support the safety of intervals greater than 2 years for these low-risk patients. While we believe these patients can be safely surveyed at a 5-year interval, the robustness of this recommendation would need to be tested in further longitudinal research.

\section{Author affiliations}

${ }^{1}$ Department of Gastroenterology and Hepatology, University Medical Center Utrecht, Utrecht, The Netherlands

${ }^{2}$ Division of Gastroenterology, Icahn School of Medicine at Mount Sinai, New York, USA

${ }^{3}$ Division of Gastroenterology and Hepatology, Vanderbilt University Medical Center, Nashville, Tennessee, USA

${ }^{4}$ IBD Clinical and Research Centre, University of Manitoba, Winnipeg, Manitoba,

Canada

${ }^{5}$ Division of Gastroenterology, Columbia University, New York, USA

${ }^{6}$ Surgical Department, Gastroenterology Division, Hospital Beatriz Angelo, Loures, Lisboa, Portugal

${ }^{7}$ Department of Gastroenterology and Hepatology, Vrije Universiteit Medical Center Amsterdam, Amsterdam, The Netherlands 
${ }^{8}$ Dutch Initiative on Crohn and Colitis (ICC), The Netherlands

${ }^{9}$ Department of Gastroenterology and Hepatology, Radboud University Medical Center, Nijmegen, The Netherlands

${ }^{10}$ Department of Gastroenterology and Hepatology, Onze Lieve Vrouwe Gasthuis Amsterdam, Amsterdam, The Netherlands

${ }^{11}$ Department of Gastroenterology and Hepatology, St Antonius Hospital

Nieuwegein, Nieuwegein, The Netherlands

${ }^{12}$ Department of Gastroenterology and Hepatology, Leiden University Medical

Centre, Leiden, The Netherlands

${ }^{13}$ Department of Gastroenterology and Hepatology, Amsterdam Medical Center,

Amsterdam, Netherlands

${ }^{14}$ Department of Gastroenterology and Hepatology, Erasmus Medical Center

Rotterdam, Rotterdam, Netherlands

Contributors JRtH, SCS: study concept and design, data acquisition, data analysis and interpretation, manuscript writing. SRS, CNB, DC, CP, EM, JE, AK, JG, AAvB, FH, JMJ, MEdJ, NM, AEvdM-dJ, CYP, CJvdW, JT: data acquisition, review of final manuscript. J-FC, TAU, SHI, BO: study concept and design, data interpretation, critical revision of the manuscript for important intellectual content.

Funding This research received no specific grant from any funding agency in the public, commercial or not-for-profit sectors.

Competing interests None declared.

Patient consent Not required.

Ethics approval Institutional Review Board at all sites.

Provenance and peer review Not commissioned; externally peer reviewed.

(c) Article author(s) (or their employer(s) unless otherwise stated in the text of the article) 2019. All rights reserved. No commercial use is permitted unless otherwise expressly granted.

\section{REFERENCES}

1 Beaugerie L, Svrcek M, Seksik P, et al. Risk of colorectal high-grade dysplasia and cancer in a prospective observational cohort of patients with inflammatory bowel disease. Gastroenterology 2013;145:166-75.

2 Lutgens MW, van Oijen MG, van der Heijden GJ, et al. Declining risk of colorectal cancer in inflammatory bowel disease: an updated meta-analysis of population-based cohort studies. Inflamm Bowel Dis 2013;19:789-99.

3 Cairns SR, Scholefield JH, Steele RJ, et al. Guidelines for colorectal cancer screening and surveillance in moderate and high risk groups (update from 2002). Gut 2010;59:666-89.

4 Annese V, Daperno M, Rutter MD, et al. European evidence based consensus for endoscopy in inflammatory bowel disease. J Crohns Colitis 2013;7:982-1018.

5 Farraye FA, Odze RD, Eaden J, et al. AGA medical position statement on the diagnosis and management of colorectal neoplasia in inflammatory bowel disease. Gastroenterology 2010;138:738-45.

6 Pinsky PF, Schoen RE, Weissfeld JL, et al. The yield of surveillance colonoscopy by adenoma history and time to examination. Clin Gastroenterol Hepatol 2009;7:86-92.

7 Robertson DJ, Burke CA, Welch HG, et al. Using the results of a baseline and a surveillance colonoscopy to predict recurrent adenomas with high-risk characteristics. Ann Intern Med 2009;151:103-9.

8 Brenner $\mathrm{H}$, Haug U, Arndt $\mathrm{V}$, et al. Low risk of colorectal cancer and advanced adenomas more than 10 years after negative colonoscopy. Gastroenterology 2010;138:870-6.

9 Singh H, Turner D, Xue L, et al. Risk of developing colorectal cancer following a negative colonoscopy examination: evidence for a 10-year interval between colonoscopies. JAMA 2006;295:2366-73.
10 Rex DK, Ponugoti PL, Johnson CS, et al. Neoplasia at 10-year follow-up screening colonoscopy in a private U.S. practice: comparison of yield to first-time examinations. Gastrointest Endosc 2018;87:254-9.

11 Atkin WS, Saunders BP. British Society for Gastroenterology, Association of Coloproctology for Great Britain and Ireland. Surveillance guidelines after removal of colorectal adenomatous polyps. Gut 2002;51(Suppl 5):v6-9.

12 Magro F, Gionchetti P, Eliakim R, et al. Third European Evidence-based Consensus on Diagnosis and Management of Ulcerative Colitis. Part 1: definitions, diagnosis, extraintestinal manifestations, pregnancy, cancer surveillance, surgery, and ileo-anal pouch disorders. J Crohns Colitis 2017;11:649-70.

13 Kappelman MD, Farkas DK, Long MD, et al. Risk of cancer in patients with inflammatory bowel diseases: a nationwide population-based cohort study with 30 years of follow-up evaluation. Clin Gastroenterol Hepatol 2014;12:265-73.

14 Carballal S, Maisterra S, López-Serrano A, et al. Real-life chromoendoscopy for neoplasia detection and characterisation in long-standing IBD. Gut 2018;67:70-8.

15 Magro F, Gionchetti P, Eliakim R, et al. Third European Evidence-based Consensus on Diagnosis and Management of Ulcerative Colitis. Part 1: Definitions, Diagnosis, Extra-intestinal Manifestations, Pregnancy, Cancer Surveillance, Surgery, and Ileo-anal Pouch Disorders. J Crohns Colitis 2017;11:649-70.

16 Claessen MM, Vleggaar FP, Tytgat KM, et al. High lifetime risk of cancer in primary sclerosing cholangitis. J Hepatol 2009;50:158-64.

17 Torres J, Pineton de Chambrun G, Itzkowitz S, et al. Review article: colorectal neoplasia in patients with primary sclerosing cholangitis and inflammatory bowel disease. Aliment Pharmacol Ther 2011;34:497-508.

18 Askling J, Dickman PW, Karlén P, et al. Family history as a risk factor for colorectal cancer in inflammatory bowel disease. Gastroenterology 2001;120:1356-62.

19 Fumery M, Dulai PS, Gupta S, et al. Incidence, Risk Factors, and Outcomes of Colorectal Cancer in Patients With Ulcerative Colitis With Low-Grade Dysplasia: A Systematic Review and Meta-analysis. Clin Gastroenterol Hepatol 2017;15.

20 Rutter MD, Saunders BP, Wilkinson KH, et al. Cancer surveillance in longstanding ulcerative colitis: endoscopic appearances help predict cancer risk. Gut 2004;53:1813-6.

21 Gupta RB, Harpaz N, Itzkowitz S, et al. Histologic inflammation is a risk factor for progression to colorectal neoplasia in ulcerative colitis: a cohort study. Gastroenterology 2007;133:1099-105. quiz 1340-1. doi.

22 Rutter M, Saunders B, Wilkinson K, et al. Severity of inflammation is a risk factor for colorectal neoplasia in ulcerative colitis. Gastroenterology 2004;126:451-9.

23 Velayos FS, Loftus EV, Jess T, et al. Predictive and protective factors associated with colorectal cancer in ulcerative colitis: a case-control study. Gastroenterology 2006;130:1941-9.

24 Lutgens M, Vermeire S, Van Oijen M, et al. A rule for determining risk of colorectal cancer in patients with inflammatory bowel disease. Clin Gastroenterol Hepatol 2015;13:148-54.

25 Flores BM, O'Connor A, Moss AC. Impact of mucosal inflammation on risk of colorectal neoplasia in patients with ulcerative colitis: a systematic review and metaanalysis. Gastrointest Endosc 2017;86.

26 Siegel RL, Miller KD, Fedewa SA, et al. Colorectal cancer statistics, 2017. CA Cancer J Clin 2017;67:177-93.

27 Nguyen GC, Bressler B. A tale of two cohorts: are we overestimating the risk of colorectal cancer in inflammatory bowel disease? Gastroenterology 2012;143:288-90.

28 Singh H, Nugent Z, Mahmud SM, et al. Predictors of colorectal cancer after negative colonoscopy: a population-based study. Am J Gastroenterol 2010;105:663-73

29 Lieberman DA, Holub JL, Morris CD, et al. Low rate of large polyps ( $>9 \mathrm{~mm}$ ) within 10 years after an adequate baseline colonoscopy with no polyps. Gastroenterology 2014;147:343-50. 